\title{
DERECHOS HUMANOS PARA LAS SOCIEDADES RURALES COLOMBIANAS
}

\author{
Elmer Castaño Ramírez \\ Profesor Titular Universidad de Caldas \\ elmercr@ucaldas.edu.co
}

Manizales, 2009-03-08 (Rev. 2009-05-10)

\section{RESUMEN}

A partir de sendas obras de Luigui Ferrajoli y Carlos Eduardo Maldonado, se describen filosóficamente los cimientos de los derechos humanos en las sociedades rurales y se dejan inquietudes que se deben investigar por quienes se inquieten por la tercera parte de nuestra población.

\section{PALABRAS CLAVE}

Derechos humanos, Sociedades rurales, Vida.

HUMAN RIGHTS FOR COLOMBIAN RURAL SOCIETIES

\begin{abstract}
Based on Luigui Ferrajoli and Carlos Eduardo Maldonado's works, the human rights foundations in rural societies are described from a philosophical standpoint. This article creates some questions that must be answered by those interested in the third segment of the Colombian population.
\end{abstract}

\section{KEY WORDS}

Human rights, rural societies, life.

\section{INTRODUCCIÓN}

Con el propósito de dejar algunas ideas de investigación en un terreno poco explorado sobre las sociedades rurales, emprendo este análisis desde Luigi Ferrajoli, filósofo contemporáneo del derecho, con especial énfasis en su libro "Los fundamentos de los derechos fundamentales"1 y, también, desde el profesor Carlos Eduardo Maldonado con su obra titulada "Hacia una fundamentación filosófica de los derechos humanos"2. Haré un recorrido sobre los cimientos, propiedades y competencias de estos derechos en las sociedades rurales para concluir con algunas inquietudes que se deben dirimir desde aquellos que se inquieten por este tema.

\section{FUNDAMENTACIÓN FILOSÓFICA}

El Derecho, con la estructura actual, nace en Roma como un mecanismo útil para certificar la propiedad de un imperio colonizador de territorios. Si bien los griegos inventan las polis como estados-ciudad, la estructura difería de la actual, la que apenas se inicia en el siglo XIX. Derecho hace alusión a lo recto, siempre en la misma dirección, pero se caracteriza, como cuerpo de reglas, por elevar a la categoría de ley la voluntad de grupos dominantes socialmente $\mathrm{y}$, además, predetermina las condiciones de vida de las sociedades mediante normas. Se trata de definir entonces cuáles son los derechos humanos y cuáles no lo son, si existen personas con más derechos que otras o con más 
derechos humanos que otras en un sentido originario y cuál es el fundamental o centro de los derechos humanos.

Si se considera la vida humana como fundamento, nos debemos ocupar entonces de determinar, en sus rasgos más esenciales y generales, lo que es ésta como cimiento de los derechos humanos. No se trata, por tanto, de una mera aprehensión de la vida, sino de su comprensión como fundamento de todo lo demás, como principio supremo y absoluto, en tanto no se disuelve y ni se explica a partir de otras ideas, sino que es ella misma fundamento de todo lo demás. En consecuencia, en los derechos humanos el interés es la vida, así como es ella misma la que hace posible todo lo demás. Más exactamente, es la vida considerada desde dos planos: desde el punto de vista de que la vida es criterio de la racionalidad del mundo, de la racionalidad de una sociedad, de un estado político o de un régimen político, y si se quiere de la racio $\neg$ nalidad de la cultura humana. En otro plano, ella define asimismo la dignidad de un grupo humano, la equidad de una acción determinada o de una serie de acciones determinadas, o inclusive, desde el punto de vista de la legitimidad de un grupo humano cualquiera, de un estado político, de un régimen político, y por qué no, de la especie humana en general. Comprender esto significa comprender la fundamentación de los derechos humanos, comprender una cimentación filosófica.

El problema axiológico en la fundamentación: si la vida humana como principio es un valor absoluto, nos tendríamos que sumergir en la axiología para definir por qué se le considera como tal. Sin embargo, surge inmediatamente una dificultad: se trata del problema de la universalidad de los valores. Cabe preguntarse con Sócrates: los valores, ¿son objetivos porque los deseamos, o bien es porque son objetivos, que los deseamos? Más específicamente, el problema de la universalidad de los valores se traduce inmediata y necesariamente como el problema mismo de la universalidad de los derechos humanos o también de la universalidad de la vida humana en la forma concreta de la universalidad de una (o varias) formas de vida bien determinadas. Los valores siempre nacen a partir de la intersubjetividad y por ello el valor es una relación y no una entidad, por ello se trata del conjunto de las relaciones (económicas, sociales, lingüísticas, sexuales) las que definen una forma de vida al interior de una sociedad, de un Estado, de una cultura, y ellas mismas los jerarquizan. La vida es el valor absoluto, en tanto que es a partir de ella que se hacen posibles todos los demás valores. A partir de la vida es posible todo lo demás, esto es todo otro valor, ideal, principio o forma de realidad. De hecho, el único absoluto es la vida, la vida humana, y ulte $\neg$ riormente también, la vida entera sobre el planeta. Ni siquiera la muerte es un valor, una alternativa a la vida, un absoluto. (Es en este punto exactamente en donde es preciso evitar malas interpretaciones acerca de una presunta incompatibilidad entre el derecho absoluto a la vida, y los problemas de la bioética, tales como la eutanasia y otros). La vida es un derecho, pero no es una obligación. La vida humana es un derecho absoluto que subsume todos los demás derechos.

La vida y el tiempo: el tiempo es una medida inventada por el hombre, basada en los aspectos físicos del planeta que habitamos y en convenciones y acuerdos pero, como medida, parte de la base del transcurrir de la vida humana. Siempre sentimos que venimos del pasado hacia un futuro, cuando no existe ni pasado, ni futuro; sólo el hoy. Pero es imposible vivir sin el pasado, sin lo que nos ha hecho (una tradición, una biografía, una historia). Sin embargo, lo esencial es aquí reconocer que, no obstante, no podemos vivir en el pasado debido a que precisamente eso equivale a negar la vida, esto es, negar las posibilidades que tenemos, las posibilidades que somos como individuos, como grupo humano, como colectividad o como especie; por tanto, no es posible vivir sin el pasado, pero la vida sí se lleva a cabo a pesar de y en contra del pasado. Es que en esencia la vida humana y por ende nosotros, somos posibilidad, posibilidad que exige realizarse, actualizarse. La vida se lanza «hacia adelante» con el pasado y al mismo tiempo en contra suyo, en contra de la historia, en contra de biografías determinadas. Decir «hacia adelante» no es, evidentemente, ningún lugar en el espacio, sino una dimensión de las vivencias y del fluir de vivencias que sí puede llegar a encarnarse en una geografía o en una coordenada determinada. $Y$ entonces, decimos, la vida se lanza, se proyecta hacia una dimensión, otra distinta del pasado, debido al pasado, y sin embargo, precisamente, a pesar suyo. 
La vida no se vive en el pasado, decimos, pero tampoco se vive sin más en el presente, puesto que el presente es fluir, transcurre incesantemente, pasa y termina sumergiéndose en el pasado; y debido a la acumulación de presentes es que vamos constituyendo el pasado; el presente termina disolviéndose acaso en la indiferencia, en la indeterminación y es el futuro, y en rigor, es (el reino de) la muerte. El presente transcurre y lo que está pasando en un rato ya sucedió y ya no tiene lugar efectivamente. Con esto se quiere decir que los derechos humanos en absoluto se enfrentan a problemas de tipo político, militar, social, etc., con una actitud como la de quien afirma "vivamos el presente", "vivamos el momento porque más adelante nadie sabe nada"; o bien, por ejemplo, con la actitud de una "defensa sin más del pasado debido precisamente a que ya fue". Quien dice "vivamos el momento porque más adelante nadie sabe" no sabe vivir, y ahí pueden esconderse o engendrarse violaciones de los derechos humanos. Más específicamente, una actitud psicológica semejante se corresponde con una realidad social y política (politeia) que engendran u ocultan un atentado a la vida y a la dignidad de las personas. De un modo equivalente, podemos sostener, o sospechar también, una violación de los derechos humanos por parte de quienes afirman la necesidad sin más del pasado, o lo que es peor, la idea de que el pasado es lo que le da sentido sin más a la vida actual de las personas, pues detrás de una afirmación semejante se esconde la negación de otras posibilidades hacia adelante, distintas de las que se simbolizan en el pasado. El Tótem es por excelencia el símbolo del pasado, y por tanto, el Tabú del futuro, de la posibilidad en cuanto tal.

Si el pasado es el reino de las cosas, el presente, por su parte, como presente puro, es la locura. En efecto, el loco vive en el presente puro, en un presente bien específico, carece de pasado y de futuro, esto es, de una perspectiva de temporalidad, está sumido tan sólo en el momento. Hablar de locura corresponde aquí al título genérico que designa el conjunto de desordenes específicamente psicóticos (y no meramente neuróticos) que pueden haber sido analizados y clasificados clínicamente o no. Con esto queremos entender comportamientos y actitudes que no se circunscriben únicamente a los muros de un hospital psiquiátrico, sino que pueden abarcar igualmente espacios más «abiertos» como la existencia de grupos humanos que viven en un presente puro o que han sido condenados a vivir el presente puro debido a estados de cosas patológicos y patologizantes. Los ejemplos en el mundo contemporáneo son variados y se encuentran en todas las latitudes. Aquí está contenido un juicio crítico acerca de formas de vida o al comportamiento y actitudes que no se limitan simplemente al individuo, primero, y segundo que no se restringen tan sólo a las esferas de la psicología clínica, de la psiquiatría o de la neurología. En esencia, la crítica se dirige longitudinalmente a una comprensión de una forma de vida, de una calidad de vida, esto es, de una dignidad humana. Pero de lo que se trata en realidad es de un análisis del fundamento de los derechos humanos: la vida, y lo que será ella.

Quien viva en el presente puro, independientemente de si ha sido objeto de análisis clínicos, vive en la locura; esto es, en la total ausencia o desprendimiento de un pasado o de una tradición, y sin ningún horizonte temporal, definido, abierto. Tal es el caso, por ejemplo, de los sectores más marginados de la sociedad, la prostitución (masculina o femenina), los enfermos terminales (S.I.D.A.) excluidos de la sociedad a la espera de que mueran, los "ancianos prematuros" (pensionados, jubilados) que no logran una readaptación a la vida social productiva y por tanto ningún sentido para vivir sumergiéndose en el pasado sin más, o en el día-a-día, los niños de los suburbios urbanos víctimas de distintas formas de violencia y maltratos y conducidos así a afrontar el día que viene ("cada día tiene su afán"), la población civil como la rural colombiana que vive en medio de guerras y que carece de un norte político, social, cultural; los pueblos que viven cercados por tropas militares, paramilitares o guerrilleras, sin ninguna certeza de lo que pueda ocurrir. Ahora bien, puesto que se trata de individuos y de grupos humanos que han sido conducidos a estados similares debido a regímenes políticos, militares, económicos, religiosos, y otros, la crítica recae, en consecuencia, sobre los agentes de estos estados de cosas. Entonces podemos hablar legítimamente de estados políticos, de situaciones de guerra, de situaciones económicas y demás que conducen a la locura, al presente puro, sin más. Se trata manifiestamente de una violación de los derechos humanos, o mejor, más radicalmente, de una violación del fundamento mismo los derechos humanos: la vida en sus propias posibilidades, la vida de las sociedades rurales, la vida de la tercera parte de la población colombiana. 
Lo que fundamenta la existencia humana, el fundamento de la dignidad, son las posibilidades que tiene, los horizontes que se plantea, las tareas que se propone y que debe poder realizar, las esperanzas que tiene, de suerte que mientras más posibilidades, horizontes, tareas y esperanzas tenga, tanto más vive; cuantas más cosas tenga por hacer y que correspondan a. sus propios proyectos, cuanto más ricos sean sus horizontes, cuantas más tareas tenga que haya que cumplir porque se siguen directamente de sus proyectos, cuantas más esperanzas racionales fundadas en un optimismo derivado de un uso adecuado de la racionalidad, tanto más pude decir que vive y tiene que vivir, que está por vivir. La vida son sus posibilidades, y ello nos permite hablar con fundamento de una vida más digna y de una calidad mejor. La calidad de vida no se limita a las condiciones exteriores, sino que incluye los propios horizontes de existencia.

La vida humana, precisamente porque es vida, es originariamente futuro, pero ese futuro debe poder cumplirse y cuando se cumple, se cumple efectivamente en tanto que presente. De tal suerte que si nos interesa el futuro no es sencillamente por el futuro mismo, lo cual sería pura utopía, sino debido a que ese futuro será y debe poder ser presente. Es precisamente esto lo que explica el hecho de que desde el punto de vista de la filosofía de los derechos humanos, el futuro sea entonces comprendido como un futuro posible, y la posibilidad como una posibilidad real. En términos más fuertes, nos interesa que el futuro se haga real. Se trata del compromiso con los propios proyectos. Una vida que no se compromete, es una vida vivida a medias. Por el contrario, una vida auténtica, radicalmente humana, es aquella que se juega en sus propias posibilidades y, dicho existencialmente, es una vida que se compromete íntegramente en sus alegrías, en sus frustraciones, en la satisfacción de las necesidades, en las de los hijos, o la familia, o los amigos, etc., es por esa razón esencial, mínima que se representa el futuro. La vida es en cuanto tiene un futuro cargado de potencialidades por llevar cabo. Lo contrario es la anormalidad dentro de la cual existe solamente la locura declarada. Aquí los derechos humanos no establecen posibilidades, tan sólo defienden los espacios para las posibilidades.

El cuidado del cuerpo: la dignidad no es un valor o una idea, sino una condición humana, donde el hombre se siente gratificado con su propia existencia y el cuerpo es el modo presente de esa existencia, en donde se depositan sus experiencias pasadas; es allí donde se deposita la historia. De ahí que el cuerpo traduce la dignidad que tiene hacia sí mismo y la que le permiten el estado político, los regímenes sociales y los económicos. Desde la escuela se desconoce el cuerpo y se le disminuye su importancia en aras a unas inteligencias (matemática, del lenguaje) no explotables como individuo en el sector rural; esto no se transcribe en niveles de nutrición, desenvolvimiento y apropiación del espacio, relaciones con las distancias, relación con otros cuerpos, relación con su propio cuerpo. No se trata de la relación de masas o con ellas, se trata de que en cada individuo hay una responsabilidad por su vida pero que además el estado debe garantizar. Un reconocimiento de las posibilidades infinitas de los individuos, choca contra las políticas y prácticas, o contra el credo y los principios de un régimen vertical. A nadie que participe en la política de defensa de los derechos humanos es desconocido este aspecto. Pero si un choque cualquiera llega a producirse, la razón para esta confrontación se encuentra en el carácter cerrado del sistema vertical del caso. Efectivamente, es propio a todo sistema vertical el asumirse como fin en sí mismo, y los individuos que se encuentran a su interior tienen tan sólo la finalidad de permitir la vida de ese sistema: de una institución, de una empresa, de una compañía o de un estado. Al sector rural se le mantiene como masa alterna para sostener las necesidades futuras del sistema económico, masa obrera de reserva para nuevos procesos productivos industriales o para la construcción urbana y, excepcionalmente, para aprovechamiento en épocas de crisis cuando se recurre a ellos porque la importación de alimentos tiene valores elevados. Es un sistema semejante el que hace por tanto, de los derechos, una cuestión peligrosa y subversiva, y nunca el contenido o los postulados mismos de los derechos del hombre o, para hablar más propiamente, de los derechos del ser humano, del hombre y de la mujer y, a partir de ellos, de los derechos sociales.

La política de los derechos humanos: la política como la voluntad de las polis es la exigencia del reconocimiento de la necesidad de la existencia humana. Luego, no es necesario solamente hablar de desapariciones, de torturas, de grupos de exterminio, etc., para hablar de los derechos humanos; no es necesario solamente hablar de magnicidios o 
de masacres, de la existencia de grupos de justicia privada y demás, para encontrar el espacio de diálogo sobre los derechos humanos que, justamente se da, por ejemplo, en la propia familia, en la vecindad, en la vereda, en la finca, en la empresa o en la iglesia. Frente a esa realidad que dice que nadie es necesario, y que ciertamente el individuo no lo es, la exigencia de la propia necesidad ya es la exigencia por una dignidad del ser humano. Lo contrario es justamente la eliminación, real o virtual, de la vida. Pues bien, es precisamente la articulación entre la dignidad de la vida humana y el reconocimiento y afirmación de su necesidad lo que hace de los derechos humanos un problema ético, además de un problema político, un problema social común.

Ahora cambiamos el panorama, ya no hablamos de derechos humanos sino de derechos fundamentales y, además, los vinculamos con las necesidades del Estado o de la República, que no es otra cosa que la diferencia romana entre derechos públicos y privados y además de la primacía del derecho público sobre el privado. Aquí se da la aparición en escena de las cuatro clases de derechos fundamentales, cada una de las cuales corresponde, como muestra el siguiente es $\neg$ quema, al producto lógico de cada clase del primer conjunto con cada clase del segundo: los derechos primarios de la persona, que son los derechos humanos; los derechos primarios del ciudadano, que son los derechos públicos; los derechos secundarios de la perso $\neg$ na, que son los derechos civiles; los derechos secundarios del ciuda dano, que son los derechos políticos.

\begin{tabular}{|l|l|l|}
\hline D. fundamentales & D. de la persona & D. del ciudadano \\
\hline D. primarios & D. humanos & D. públicos \\
\hline D. secundarios & D. civiles & D. políticos \\
\hline
\end{tabular}

Para aclararlos vale el siguiente esquema:

\section{LOS FUNDAMENTOS DE LOS DERECHOS FUNDAMENTALES}

\begin{tabular}{|l|l|l|l|}
\hline $\begin{array}{l}\text { Derechos (D). } \\
\text { fundamentales }\end{array}$ & \multicolumn{2}{|c|}{ D. de la persona ciudadano } \\
\hline $\begin{array}{l}\text { D. primarios (sólo } \\
\text { expectativas- } \\
\text { sustanciales) }\end{array}$ & $\begin{array}{l}\text { D. de libertad } \\
\text { (expectativas. } \\
\text { negativas.) }\end{array}$ & $\begin{array}{l}\text { libertad de(prensa) } \\
\text { asociación reunión) }\end{array}$ & $\begin{array}{l}\text { D. públicos (expectativas } \\
\text { positivas: Salud, } \\
\text { educación, seguridad } \\
\text { social) }\end{array}$ \\
\hline & & libertad para (vivir) & \\
\hline $\begin{array}{l}\text { D. secundarios } \\
\text { (expectativas. y } \\
\text { poderes- formales) }\end{array}$ & D. civiles (D. de autonomía privada) & $\begin{array}{l}\text { D. políticos (D. de } \\
\text { autonomía política) }\end{array}$ \\
\hline
\end{tabular}

El verdadero problema, de carácter filosófico-político o de teo 7 ría de la justicia, es el del fundamento axiológico de los derechos fundamentales. La cuestión es la mirada clásica de carácter ético-político expresada en la pregunta ¿qué derechos deben ser (o es justo o está justificado que sean) tutelados como fundamentales? y admite, por tanto, una respuesta de tipo no asertivo sino normativo, cuya funda 7 ción racional exige la formulación de los criterios meta-éticos y meta-políticos idóneos para justificar su estipulación normativa, confor $\neg$ me a los fines o los valores ético-políticos que aquéllos sean capaces de satisfacer. Es posible identificar cuatro criterios axiológicos, todos ellos referidos al valor de la persona humana asumida como fin y nunca como medio, según la clá sica máxima de la moral kantiana. Estos criterios sirven, por un lado, para determinar las opciones ético-políticas en favor de los valores de la persona — vida, dignidad, libertad, supervivencia- que son establecidos positivamente como funda 7 mentales bajo la forma de expectativas universales; y por otro, son todos sugeridos por la experiencia histórica del constitucionalismo democrático, tanto estatal como internacional, y que, finalmente, ha resultado de hecho, orientada por ellos. 
Los derechos civiles de adquirir y disponer de bienes de propiedad y los derechos patrimoniales adquiridos a través de su ejercicio como los derechos reales de propiedad, son singulares y disponibles. La indisponibilidad de los primeros es, en realidad, el presupuesto de la disponi $\neg$ bilidad de los segundos, se someten a la dinámica del mercado, se basan en él. Pero precisamente por ello — porque son dere 7 chos-poder cuyo ejercicio produce efectos obligatorios desiguales y virtualmente ilimitados sobre las libertades propias y las de los de $\neg$ más-, los derechos civiles no pueden dejar de estar sometidos a la ley, ni por tanto ser confundidos con los derechos de libertad que, por el contrario, representan para aquéllos un límite infranqueable. Ya que en los inicios del derecho el Estado garantizaba los límites del derecho, hoy el Estado, lamentablemente, sólo garantiza los límites del mercado, muchísimo más visibles en la propiedad rural.

Se debe afrontar el problema de los cimientos de los derechos fundamentales aclarando y distinguiendo los diversos significa 7 dos que pueden asociarse a la palabra «fundamento». Estos signifi $\neg$ cados son cuatro: a) la razón o fundamento teórico; b) la fuente o fundamento jurídico; c) la justiาficación o fundamento axiológico; d) el origen o fundamento histó $r$ rico y sociológico. Son, correlativamente, diferentes los significados intencionales del adjetivo "fundamentales» asociado a «derechos»: según que hagan referencia a su forma universal en el plano de la teoría del derecho, a las constituciones o leyes fundamentales que los recogen en el plano jurídico, a los valores asumidos como básicos en el plano axiológico, o a los procesos políticos y sociales mediante los cuales son promovidos e implementados en el plano histórico o sociológico.

El primero de estos criterios es el del nexo entre derechos funda 7 mentales e igualdad. Es claro que no existen las mismas igualdades, y antes por el contrario existen niveles de igualdad, lo que crea grandes desigualdades, la forma universal de tales derechos equivale a la igualdad en la titularidad de los derechos de los sujetos -perาsonas o ciudadanos capaces de obrar- a los que les son atribuildos. Es preciso, obviamente, determinar "en qué» -la vida, ciertas libertades, las necesidades vitales- es justo o está justificado el que los derechos aseguren esta igualdad: es ésta, precisamente, la determinación axiológica que hace de la igualdad un criterio para identificar de cuáles «deben ser» los derechos que merecen ser tutelados como fundamentales. El segundo criterio, en conexión con el primero, está entre derechos fundamentales y democracia. Los vínculos impuestos a la mayoría por los derechos fundamentales llamados la «dimensión sustancial» de la democracia -derechos políticos, civiles, de libertad y sociales- servirán para configurar la base de otras tantas dimensiones axiológicas de la democracia. El tercer criterio es el del nexo entre derechos fundamentales establecidos en el preámbulo de la Declaración Universal de 1941. Deben garantizarse como derechos fundamentales todos los derechos vitales cuya garantía es condición necesaria de la convivencia pacífica: el derecho a la vida y a la integridad personal, los derechos de libertad, los civiles y políticos pero, también, en un mundo en que sobrevivir es un hecho cada vez menos natural y progresivamente más artificial, los derechos sociales a la supervivencia. El cuarto criterio es el papel de los derechos fundamentales como leyes del más débil. Con esta base, todos los derechos funda 7 mentales -del derecho a la vida, a los derechos de libertad y los derechos sociales- pueden ser definidos, en el plano axiológico, como leyes del más débil en alternativa a la ley del más fuerte que imperaría en su ausencia: de quién es más fuerte físicamente; de quién es más fuerte polínticamente, como en el Estado absoluto; de quién es más fuerte eco $n$ nómica y socialmente, como en el mercado capitalista.

La clase de sujetos de los que es predicable la igualdad sería acaso la «Persona». Ésta, en el plano de la teoría del dere $\mathrm{ch}$, no equivale a «ser humano», sino más bien al status de los sujetos, cualesquiera que sean, normativamente previstos como pre supuesto de situaciones jurídicas, y específicamente, de los «dere $\urcorner$ chos de la persona». La equivalencia entre «persona» y «ser humaาno» y la consiguiente correlación entre «igualdad en los derechos» y "persona humana» se han afirmado, sin embargo, no sólo en el plano axiológico, sino también en el plano del derecho positivo con el reconocimiento del status de «persona» a todos los seres humannos, producido con las primeras codificaciones y con la configura $\urcorner$ ción, en las constituciones modernas, de casi todos los derechos fundamentales -desde los derechos de libertad a los derechos civi-les, hasta gran parte de los derechos sociales- como "derechos de la persona», y sólo de los derechos políticos y de algunos derechos sociales como «derechos del ciudadano». 
Dentro del problema de la democracia, ésta se debería entender como la delegación que hago mediante mi voto de mi capacidad de administrar, pero no para que se haga lo que se venga en gana, sino para que se me consulte. La democracia no es sólo un método, sino también un ideal: el ideal igualitario. Donde este ideal no inspira a los gobernantes de un régimen que se llama democrático, la demo 7 cracia es un nombre en vano. No se puede separar la democracia for $\urcorner$ mal de la sustancial. Donde sólo existe la primera, un régimen democrático no está destinado a du rar. Por lo demás, si históricamente el derecho moderno ha naci $\neg$ do de la afirmación de la primacía de los derechos sobre los deberes, la democracia moderna ha nacido de la afirmación de la primacía de los derechos sobre los poderes, esto en un doble aspecto: en cuanto a las modalidades de legitimación formal del poder, confiada a los derechos secundarios de autonomía políti $\neg$ ca y civil; y en cuanto a los contenidos a los que el ejercicio del poder queda vinculado, confiados, a su vez, junto a su legitimación sustancial al respeto de los derechos primarios de libertad y sociales. Si, por consiguiente, entendemos el término «pue $\neg b l o »$, a falta de algo mejor, en el sentido de «todos», la democracia (formal) resulta ser, en el primer aspecto y gracias a los derechos secundarios, el poder de todos. En el segundo aspecto, a su vez, la democracia (sustancial) es, gracias a los derechos primarios, el contrapoder de todos, es decir, el sistema de límites y vínculos opuesto por todos, a sí mismos, en garantía de todos. Es cierto, por otro lado, que los derechos fundamentales no suponen ni requieren el consenso de todos, pero si en decisiones trascendentales como la guerra.

Es evidente, en efecto, que la implicación recíproca entre paz y tutela de los dere 7 chos humanos supone que ninguna tutela de los derechos puede ser realizada mediante la guerra, antes que con el derecho. En primer lugar, porque la guerra, y tanto más la guerra en el campo que afecta casi exclusivamente a las poblaciones civiles que habitan el territorio donde se desenvuelve el sector agropecuario, supone, por naturaleza, una negación del derecho y de los derechos, una violación en masa del derecho a la vida de millares de inocentes que nada puede justifficar, una violencia descontrolada cuyos efectos destructivos son, como ha confirmado la guerra intestina colombiana, diametralmente opuestos a los fines perseguidos. En segundo lugar, porque si se desvanece el nexo entre medios y fines, entre derecho y derechos, entre forma y sustancia de su tutela, los derechos fundamentales se resuelven en una nueva enésima abstracción, en perjuicio de las personas de carne y hueso que son sus titulares; el equivalente moderno del «bien» que ha justificado las guerras en todos los tiempos, corren el riesgo de desacreditarse como la consigna de un nuevo tipo de fundamentalismo que contrapone Occidente al resto del mundo, según la lógica identitaria de las guerras étnicas: de un lado, Occidente, del otro, el mundo restante al que Occidente pretende imponer, con la violencia, sus propios valores; de un lado los buenos del sector urbano contra los malos de las selvas y el sector rural quienes deben someterse para seguir sosteniendo los beneficios del primero; por una parte los que creen en el bienestar para todos y de la otra, los que valoran sólo a quienes tienen poder y dinero lo que les da más derechos y, además, derechos sobre los demás.

La ciudadanía es violada por nuestras legislaciones en materia de extranjeros, inmigrantes y desplazados. Si acaso, son «normati-vas» y "axiológicas» (en sentido regresivo) respecto al derecho vingente, las tesis opuestas que -en contraste con lo que dicen códigos civiles, constituciones estatales y cartas internacionales- «propornen», precisamente, que la ciudadanía sea considerada como el «con-tenedor» de todos los derechos, o bien que «el concepto de derechos humanos» sea circunscrito sólo al de derechos del ciudadano. Una cuestión distinta, ésta sí de carácter axiológico, es la pers $\neg$ pectiva de una ciudadanía universal o, lo que es lo mismo, la aboliาción de las ciudadanías estatales como presupuesto de los derechos políticos que bien podrían asociarse a la simple residencia. De los dos status, junto al de «perso $\neg n a »$, la capacidad de obrar y la ciudadanía, el primero es básico para todos y de cualquier manera insuprimible como presupuesto de los dere $\urcorner$ chos-poder que son los derechos de autonomía, el segundo designa, en realidad, un accidente «de nacimiento» no sólo superable sino también injustificable en el plano ético-político. Pero esta es una cuestión secundaria respecto al problema político -no axiológico- de la superación de los vínculos jurídicos y de hecho que, en contradicción con el artículo 2 de la Declaración de 1948 y con las propias constituciones estatales, condicionan de manera ile $\operatorname{cítima~no~sólo~los~}$ derechos del ciudadano, sino también los derechos de la persona, al «origen nacional» de sus titulares, expresado preciาsamente por la ciudadanía. Ésta es la antinomia regresiva entre dere $\urcorner$ chos universales y ciudadanía, destinada a tornarse explosiva con el 
crecimiento de las desigualdades y las presiones migratorias como fuente permanente de peligros para la paz y para la credibilidad misma del derecho, tanto interna $c i o n a l$ como constitucional. Los desplazamientos y migraciones intestinas contienen flagrantes pérdidas de derechos civiles y patrimoniales, producto de fuerzas paramilitares, guerrilleras o de delincuencia común, desde donde no se cuenta con protección del Estado y, antes por el contrario, se justifican políticamente desde el bien común citadino en aras a mantener la guerra en la periferia (rural). Aquí no sólo se deja de ser persona, ciudadano, sino, en algunos casos, ser humano sujeto de derechos; pasan a convertirse en individuos solamente dignos de los derechos a la misericordia estatal o a la benevolencia de patrones que se lucran de esa masa informe o sujetos simplemente de caridad pública.

Es necesario reiterar que las repúblicas y Estados no son sinónimos de orden, por el contrario, en ellos la desigualdad es legitimada por la ideología liberal según la cual la autonomía empresarial no es un poder, sujeto en cuanto tal al derecho, sino una libertad, y el mercado necesita, para producir riqueza y ocupación, no reglas, sino no ser sometido a ningún límite ni a ninguna regla. Ideas que son contrarias al modelo normativo del Estado constitucional de derecho, insostenibles en el plano económico, ya que ningún mercado puede sobrevivir sin reglas y sin intervenciones públicas dirigidas a garantizar su respeto. Por lo demás, estas intervenciones no faltan, sólo que se dan de forma prevalente en favor de los países más ricos y de las grandes empresas. Piénsese en las políticas del Banco Mundial y del Fondo Monetario Internacional en materia de deuda externa y de políticas universales que condicionan las decisiones internas, homogeneizándolas a favor de intereses supranacionales y en contravía de culturas, historias, ambientes ecológicos, responsables del aumento del hambre, la miseria y las enfermedades de las que son víctimas en el mundo millones de seres humanos.

El papel garantista del Estado es hoy impuesto por el carácter supra-estatal que las declaraciones y convenciones interna $\urcorner$ cionales confieren a los derechos fundamentales, en modo sólo com?parable a la escala planetaria alcanzada por los máximos poderes, tanto público como privado. Ha entrado en crisis, con el viejo Estado Nacional, el tradicional sistema de fuentes del derecho, fun $\urcorner$ dado en la unidad, la coherencia y la plenitud de los ordenamientos estatales. Ello ha provocado una creciente incertidumbre generada, como en el derecho pre-moderno, por la superposición de fuentes y de ordenamientos concurrentes, además de un debilitamiento del constitucionalismo y del garantismo a causa del desplazamiento de los poderes de decisión hacia organismos supra-estatales que, de he $c h o$, deciden sin responsabilidad política y sin límites constituciona 7 les. Piénsese en la Unión Europea que, con todo, representa sin duda el caso de integración regional más democrático y progresista. Al desplazar, fuera de los límites de los Estados Nacionales los ámbitos y formas de decisión tradicionalmente reservados a su soberanía, el proceso de integración europea está, por ahora, poniendo en crisis la tradicional jerarquía de fuentes y reduciendo, en ausencia de con $\neg$ troles parlamentarios y jurisdiccionales de constitucionalidad, el papel garantista de las constituciones nacionales. Más aún, piénsese en los espacios de poder neo-absolutista que se han abierto en obligaciones inherentes a las relaciones de trabajo rural y a la tutela de los consumidores, tan pobre en los dispendios colombianos. Finalmente, las distintas formas de incompatibilidad entre los poderes públicos y privados, forman parte del constiᄀtucionalismo profundo de toda democracia, basado ante todo en la separación entre la esfera pública y la privada y en la inadmisibilidad de conflictos de intereses entre ambas esferas. Estos límites y víncuᄀlos, no siempre establecidos eficazmente por los derechos estatales, son hoy arrasados por el nuevo "derecho de la globalización», mode $\neg$ lado no ya sobre la ley sino sobre la contratación, por tanto, existe un sustancial vacío de dere 7 cho que abre espacios incontrolados a la explotación del trabajo y del medio ambiente, a las diversas formas de criminalidad económi $\urcorner c a$ y a las correspondientes violaciones de derechos humanos, cada vez más evidentes en el sector rural.

La globalización de la economía, en ausencia de reglas, ha proาvocado de este modo un crecimiento exponencial de las desigualda $\neg$ des: el aumento de las riquezas en nuestras democráticas sociedades opulentas y, junto a ello, de la pobreza, el hambre y la explotación en el resto del mundo. Se ha calculado que la brecha de ingreso entre los países más pobres y los más ricos del planeta (que era de 1 a 3 en 1820, de 1 a 11 en 1913 y de 1 a 35 en 1950) se ha vuelto de 1 a 72 en 1992, y que menos de trescientos 
millonarios (en dólares) poseen más riqueza que la mitad de la po $\neg$ blación mundial, es decir, que tres mil millones de personas. Más exactamente la diferencia de ingreso entre el quinto de la población mundial que vive en los países más ricos y el quinto que vive en los países más pobres era de 30 a 1 en 1960, de 60 a 1 en 1990, y de 74 a 1 en 1997 . Se ha calculado, además, que el patrimonio de las tres personas más ricas del mundo supera la suma del producto nacional bruto de todos los países menos desarrollados y de sus 600 millones de habitantes; y que una contribución anual equivalente a $1 \%$ de la riqueza de las 200 personas más ricas del mundo, cuyo patrimonio es superior al ingreso conjunto de $41 \%$ de la población mundial, bastaría para asegurar el acceso universal a la educación primaria para todos. Aunque la producción per capita de alimentos ha experimentado un incremento de 25\%, cerca de 840 millones de personas están desnutridos y casi mil millones no pueden acceder a los alimentos básicos; además, pese al incre $\urcorner$ mento global en la asistencia sanitaria, de la tasa de escolarización y de la esperanza de vida y, sumándole a ello la fuerte reducción total de combustibles altamente contaminantes, se ha duplicado el número de enfermos de sida, 850 millones de personas son analfabetas, 3 millones mueren cada año como consecuencia de la contaminación atmosférica y más de 5 millones por causa de la contaminación del agua. Estimaciones de la Organización Mundial para la Salud señalan que en el mundo actualmente ocurren 3 millones de intoxicaciones agudas por plaguicidas con 220.000 casos fatales (Ulloa S., 1998). Lo anterior se debe no sólo a la utilización de una tecnología (principalmente para agricultura) intrínsecamente peligrosa y a la falta de un derecho protector escasamente representado en algunas normas desconocidas por los productores y sin vigilancia ni protección real del Estado, sino también a la demostración del estado mismo de la importancia de mantener los negocios de la química como la única estrategia productiva agraria en un país como el nuestro; bajo estas circunstancias ¿será posible la vida y la dignidad humana sobre el planeta?

Investigaciones necesarias sobre las sociedades rurales: con esta visión se abren grandes y urgentes campos de investigación sobre las sociedades rurales, que en Colombia se conforman por comunidades indígenas variadas, etnias de descendencia afro, grupos desprotegidos de agricultores y pequeños productores, comunidades urbanas de servicios rurales y grandes agricultores. Así entonces, ¿cómo diferenciar lo público, privado e íntimo, mediado el uso de la fuerza estatal con fumigaciones aéreas sobre pobladores del campo?, ¿cuáles son los derechos que favorecen el potencial de futuro de los niños que habitan el campo como futuros ciudadanos no mendicantes para éste país?, ¿cuáles son las actividades garantistas del Estado colombiano sobre la tercera parte de la población colombiana que ocupa el territorio rural?, ¿son personas para efectos jurídicos los pequeños agricultores con pobres niveles de escolaridad?, ¿hay igualdad frente a los derechos cuando no se dispone de condiciones mínimas de sobrevivencia?, ¿quién compensa las violaciones del derecho a la vida, a la propiedad, a la libertad sobre los ocupantes del territorio rural?, ¿cómo piensan sus valores las comunidades desplazadas por la violencia en el campo?, ¿cómo prospectan su futuro las comunidades con desnutrición crónica y sin garantías de sobrevivencia?

\section{BIBLIOGRAFÍA}

- $\quad$ FERRAJOLI L. Los fundamentos de los derechos fundamentales segunda edición 2005 Ed. Trotta SA.

- MALDONADO C.E. Hacia una fundamentación filosófica de los derechos humanos Arango Editores Santa fé de Bogotá 1999

- ULLOA S. Y. Prevención y tratamiento de intoxicaciones agudas por compuestos organofosforados en la atención primaria de salud. La Habana: Ediciones Finlay, 1998 


\section{NOTAS}

1. Ferrajoli L. Los fundamentos de los derechos fundamentales segunda edición 2005Ed. Trotta S.A.

2. Maldonado C.E. Hacia una fundamentación filosófica de los derechos humanos Arango Editores Santa fé de Bogotá 1999 